\title{
Comparison of predictor approaches for longitudinal binary outcomes: Application to anesthesiology data
}

Longitudinal data with binary repeated responses are now widespread among clinical studies and standard statistical analysis methods have become inadequate in the answering of clinical hypotheses. Instead of such conventional approaches, statisticians have started proposing better techniques, such as the Generalized Estimating Equations (GEE) approach and Generalized Linear Mixed Models (GLMM) technique. In this research, we undertook a comparative study of modeling binary repeated responses using an anesthesiology dataset, which has 375 patient data with clinical variables. We modeled the relationship between hypotension and age, gender, surgical department, positions of patients during surgery, diastolic blood pressure, pulse, electrocardiography and doses of Marcain-heavy, chirocaine, fentanyl, and midazolam. Moreover, parameter estimates between the GEE and the GLMM were compared. The parameter estimates, except timeafter, Marcain-Heavy, and Fentanyl from the GLMM, are larger than those from GEE. The standard errors from the GLMM are larger than those from GEE. GLMM appears to be more suitable approach than the GEE approach for the analysis hypotension during spinal anesthesia. 


\section{Authors:}

3 Anil Aktas Samur*1, Nesil Coskunfirat ${ }^{2}$, Osman Saka $^{1}$

\section{Affiliations:}

$5 \quad{ }^{1}$ Akdeniz University, Faculty of Medicine, Department of Biostatistics and Medical Informatics, 6 Antalya, Turkey

$7{ }^{2}$ Akdeniz University, Faculty of Medicine, Department of Anesthesiology and Reanimation, 8 Antalya, Turkey

\section{$9 *$ Corresponding author}

10 Anil AKTAS SAMUR, PhD

11 Department of Biostatistics and Medical Informatics

12 Akdeniz University

13 Faculty of Medicine

14 07059, Antalya, Turkey

15 +1 (617) 794-0836

16 E-mail: anilim@gmail.com 
18 Longitudinal studies are designed to evaluate change within an individual over time. Repeated observations and covariates are conducted with these individuals. Because repeated measurements are made on the same subjects at different times, multiple assessments within subject responses are positively correlated. In analyzing longitudinal studies, this dependence must be accounted for in order to make correct inferences (Fitzmaurice \& Laird 1993; Fitzmaurice et al. 1993; Laird \& Ware 1982).

24

Several models have been proposed for the analysis of clustered data. A particular feature of longitudinal data is that they are clustered. The dependent variable is measured for each subject, and the subjects belong to a cluster, such as families, or classes. In longitudinal studies, the dependent variable is measured repeatedly for the same subject on different occasions, and subjects are clustered within the same unit. The dependent variables within the same cluster are assumed to be correlated (Agresti 2002; Fitzmaurice et al. 2004).

Most of these models are extensions of the generalized linear models with logistic, probit, or complementary log-log link functions (Carriere \& Bouyer 2002). These link functions are used for binary dependent variables. These models are usually classified into marginal or random effects models. Marginal models are also called population-averaged models, whereas randomeffects models are also referred to as generalized linear mixed models (GLMM), or multilevel models.

In a marginal model, the entire response vector is modeled marginally on a set of covariates; the association structure is then typically captured via a set of association parameters, such as correlations, odds ratios, etc. The marginal model for the mean response depends only on the covariates of interest, not on any random effects or previous responses.

The generalized estimating equation (GEE) approach is the most popular method seen in marginal models. GEE is an extension of generalized linear models (GLM) for the analysis of longitudinal data. In this method, the correlation between measurements is modeled by assuming a working correlation matrix. This assumption eases the estimation of model parameters. Estimating the correct working correlation matrix provides efficiency parameter estimates. Even if it isn't correctly estimated, the model parameters from GEE tend to be consistent (Hardin \& Hilbe 2007).

Moreover, GLMM is an extension of GLM, inasmuch as it allows random effects in linear predictors. GLMM is useful for modeling the dependence among response variables in longitudinal or repeated measures studies, as well as for accommodating over-dispersion among responses. Over-dispersion refers to the presence of higher variability than expected in dataset. Over-dispersion may occur when assuming that a dependent variable has binomial distribution in GLMMs. This is because variance is a function of the mean for binomial distribution. It occurs when there is a correlation between observations, or observations are collected from clusters, or due to the heterogeneity of the subjects. 
55 In GLMMs, the model is constructed with both a fixed and a random component. The fixed 56 component usually estimates the experimental effect, whereas the random component estimates 57 the heterogeneity across clusters in the regression coefficient (Moscatelli et al. 2012).

58 The aim of this study was to compare the regression parameters and standard error of two 59 analyses for longitudinal data. Additionally, in this paper we investigated the GEE and the 60 GLMM approaches for predictor analysis in order to identify factors associated with hypotension 61 during the intra-operative and post-operative period.

62

63

64

65

66

67

68

69

\section{Generalized Estimating Equations (GEE)}

The generalized estimating equations (GEE) approach proposed by Liang and Zeger is, as previously noted, an extension of generalized linear models (GLM). GLM is a linear model and in the GLMs, the response variable has a distribution pattern seen in the exponential family. A GLM can be defined by three components. The first is the linear predictor $\eta$, which is a linear combination of regression coefficients;

$$
\eta_{i}=x_{i}^{\prime} \beta
$$

The second is the link function $g($.$) that relates the mean of the data to the linear predictor;$

$$
\begin{gathered}
Y_{i} \\
E(\dot{i}) \\
\dot{i} \\
g i
\end{gathered}
$$

The last component is the response distribution for $Y_{i}$ from the exponential family of distributions (Agresti 2002; Mccullagh \& Nelder 1989).

The GEE approach is used for the analysis of correlated response data (Dahmen \& Ziegler 2004; Liang \& Zeger 1986; Omar et al. 1999). This method does not require distributional assumptions. GEE describes changes in the population mean and is used to estimate population average models or marginal models (Fitzmaurice et al. 2008). An advantage of this approach is that if the model for the mean has been correctly specified, consistent estimators can be obtained, even if other components of the model, such as the working correlation matrix, have been mis-specified (Hardin \& Hilbe 2003; Warton 2011).

Let $y_{i}=\left(y_{i 1}, \ldots, y_{i j}, \ldots, y_{i n_{i}}\right)^{T}$ represent the response vector for the $i$-th subject, where we assume that observations from the same subject are correlated or depend on each other to some extent. Observations from different subjects are assumed to be independent. The observed value $y_{i j}$ is related to the linear predictor $x_{i j}^{T} \beta$ towards the appropriate link function,

$$
g\left(E\left(y_{i j}\right)\right)=x_{i j}^{T} \beta
$$


82 where $\mathrm{g}$ is an appropriate link function, which identifies a function of the mean that is a linear 83 prediction of covariates, e.g. identity for continuous response variables, or the logit function for 84 binary response, and $\beta$ is a vector of regression coefficients. The variance is defined by

$$
\operatorname{var}\left(y_{i j}\right)=\phi V\left(E\left(y_{i j}\right)\right)
$$

85

86

87 with $V_{i}=\square A_{i}^{\frac{1}{2}} R_{i}(\alpha) A_{i}^{\frac{1}{2}}$, where

88

89

90

91

92

93

94

95

96

97

98

99

100

101

102

103

104

105

106

$$
\sum_{i=1}^{N} \frac{\partial \mu_{i}}{\partial \beta^{T}} V_{i}^{-1}\left(Y_{i}-\mu_{i}\right)=0
$$
GLMM:

where $V$ is a known variance function and $\phi$ is a possible unknown scale or over-dispersion parameter. The regression coefficient estimates, $\beta$ are defined by the solution of the GEE

as the $t$-th diagonal element and $R_{i}(\alpha)$ is the working correlation matrix of $Y_{i}$, indexed by a vector of parameters $\alpha$ (Dahmen \& Ziegler 2004; Fitzmaurice et al. 2008; Kopcke et al. 2004; Liang \& Zeger 1986; Omar et al. 1999).

\section{Generalized Linear Mixed Models (GLMM)}

The Generalized Linear Mixed Model (GLMM) is an extension of the GLM for clustered categorical data. The GLMM combines two statistical frameworks, which are the GLM and Linear Mixed models (LMM). GLMs combine regression models for different response types such as linear models for continuous responses, logistic models for binary responses, and loglinear models for counts. LMMs are linear regression models that include normally distributed random effects in addition fixed effects (Fitzmaurice et al. 2008).

In the LMM, it is assumed that the conditional distribution of each $Y_{i j}$, given a vector of random effects $b_{i}$, has a normal distribution, with $\operatorname{Var}\left(Y_{i j} \mid b_{i}\right)=\sigma^{2}$. Furthermore, given the random effects $b_{i}$, it is assumed that the $Y_{i j}$ are independent of one another (given $b_{i}, Y_{i j}$ and $Y_{i k}$ are assumed to be independent of each other) (Fitzmaurice et al. 2004).

In the GLMM, it is assumed that the conditional distribution of each $Y_{i j}$, given a $q \times 1$ vector of random effects $b_{i}$, belongs to the exponential family of distributions (Fitzmaurice et al. 2004). The GLMM uses the inverse link function to describe the relationship between the linear predictor and the conditional mean (Katrien \& Jan 2005). The linear predictor for the

$$
g\left(E\left(Y_{i j} \mid b_{i}\right)\right)=\eta_{i}=X_{i j}^{\prime} \beta+Z_{i j}^{\prime} b_{i}
$$

107 where $g($.$) is a known link function. The variance of each of Y_{i j}$, given a vector of random 108 effects $b_{i}$,

$$
\operatorname{Var}\left(Y_{i j} \mid b_{i}\right)=v\left\{E\left(Y_{i j} \mid b_{i}\right)\right\} \phi
$$


109 where $v($.$) is a known variance function, a function of the conditional mean E\left(Y_{i j} \mid b_{i}\right)$ 110 (Fitzmaurice et al. 2004). Also, given the random effects $b_{i}$, it is assumed that the $Y_{i j}$ are 111 independent of one another; this is the so called "conditional independence" assumption 112 (Fitzmaurice et al. 2008). The random effects are assumed to have some probability distribution. 113 Any multivariate distribution can be assumed for the $b_{i}$; in practice it is common to assume 114 that $b_{i}$ have a multivariate normal distribution, with zero mean, and $q \times q$ covariance 115 matrix, G. In addition, the random effects, $\quad b_{i}$ are assumed to be independent of the covariates, $116 X_{i} \quad$ (Fitzmaurice et al. 2004).

117 The GLMMs are the GLMs that include multivariate normal random effects in the linear 118 predictor. Nevertheless, there is a difference between the GLM and the GLMM; this difference is 119 error terms. The GLM with probit link function is:

$$
\begin{gathered}
Y_{i j}=1 \\
P i=\beta_{0}+\beta_{1} x_{i j} \\
\Phi^{-1} i
\end{gathered}
$$

120 Latent variable $Y^{i}{ }_{i j}$ and the model is defined as;

$$
Y_{i j}^{i}=\beta_{0}+\beta_{1} x_{i j}+v_{i j}
$$

121 The error term $v_{i j}$ is the sum of two error terms, such that:

$$
\begin{aligned}
& v_{i j}=u_{i}+\varepsilon_{i j} \\
& u_{i} N\left(0, \sigma_{u}^{2}\right) \\
& \varepsilon_{i j} N\left(0, \sigma_{\varepsilon}^{2}\right)
\end{aligned}
$$

122 The error term $\varepsilon_{i j}$ represents the variability within subjects and the other error term $u_{i}$ 123 represents the variability between subjects. Additionally, the error term $u_{i}$ is also known as the 124 random effects parameter (Moscatelli et al. 2012).

125 In GLMMs the overall variability is separated into a fixed and a random component. The fixed 126 component usually estimates the effect of interest, such as the experimental effect, whereas the 127 random component estimates the heterogeneity between clusters (i.e., between subjects) 128 (Moscatelli et al. 2012). The GLMM is used to analyze changes in individual response means, 129 rather than population average. This model is therefore appropriate for modeling and for the 130 prediction of individual response profiles.

\section{Description of the Clinical Data}

132

133

134

135

136
All of the cases that were admitted to the Akdeniz University Hospital Anesthesiology and Reanimation Department during the period of January 2008 to January 2011 were evaluated retrospectively. The records of 417 patients who had spinal anesthesia within this 3 year time period were obtained. Patients below 17 years old were excluded. 375 of those 417 patients were over 17 and were therefore included in the study. 
137 Hypotension is common during spinal anesthesia (Sharma et al. 1997). According to the 138 literature, hypotension has an incidence of 15\% to 33\% (Carpenter et al. 1992; Hartmann et al. 139 2002; Lin et al. 2008). Certain studies have shown that people who receive anesthesia during the 140 operation can die as a result of hypotension. According to studies defining the factors associated 141 with hypotension, there are particular risk factors, such as age, gender, anesthesia drugs and 142 doses (Carpenter et al. 1992; Hartmann et al. 2002; Maxson 1933; Tarkkila \& Kaukinen 1991).

143 The outcome variable of interest in our study was hypotension. Interestingly, there is no 144 universally accepted definition of hypotension in the literature. In a systematic review, Klöhr et.al 145 highlighted the two most frequently used definitions of hypotension. Based on Klöhr et. al. 146 (2010), we have therefore used the following definition, which is systolic blood pressure $(\mathrm{SBP})<$ $147100 \mathrm{mmHg}$, or a decrease of $<80 \%$ of the baseline SBP, to define hypotension.

$$
\text { Hypotension }=\left\{\begin{array}{c}
1(\text { yes }), \text { if } S B P<100 \vee S B P<(\text { baseline } S B P) * 0.8 \\
0(\text { no }), \text { if } S B P \geq 100 \vee S B P>(\text { baseline } S B P) * 0.8
\end{array}\right\}
$$

148 Our independent variables were:

149

150

151

152

153

154

155

156

157

158

159

160

161

162

163

164

165 166

167

i. Patient's age (year);

ii. Patient's gender: Male or Female

iii. Surgical department: general surgery, urology, obstetrics and gynecology (O\&G)

iv. Positions of patients during surgery: Lithotomy, supine

v. Diastolic blood pressure (DBP)

vi. Pulse

vii. Dose of Marcain-heavy

viii. Dose of Chirocaine

ix. Dose of Fentanyl

x. Dose of Midazolam

xi. Electrocardiography (ECG)

There was no missing data in either the outcome or the covariates. The outcome variable was hypotension that had been diagnosed within a 40-minute period. Hypotension was recorded every 5 minutes during the surgery. Figure 1 shows the change in the probability of hypotension at each 5-minute interval.

(Figure 1)

The mean (SD) age was 48.81 years (18.91) and the summary statistics (median, and IR (interquartile range) for the doses of anesthetic drugs are given in Table 1.

(Table 1)

168 In this study, $56 \%(n=210)$ of patients were male, $44 \%(n=165)$ were female. $38.4 \%(n=144)$ of 169 patients underwent surgery at the department of obstetrics and gynecology, 44\% $(\mathrm{n}=165)$ at the 170 urology service, and $17.6 \%(n=66)$ underwent surgery at the general surgery service. In $41.9 \%$ 
$171(n=157)$ of patients, surgery was performed in the lithotomy position, while $58.1 \%(n=218)$ of

172 patients were placed in the supine position. In $97.6 \%(n=366)$ of patients ECG was normal; in

$1732.4 \%(\mathrm{n}=9)$ of patients it was abnormal. The summary statistics of DAP and Pulse are given in

174 Table 2.

175

(Table 2)

176 In figure 1, the probability of hypotension does not change regularly over time. There are 177 different slopes. For this reason, it is assumed that every patient has a two-piece linear spline 178 growth curve with a knot at the time of surgery. Piecewise regression was used to define a 179 breakpoint for our study (BƯrŽKOVÁ \& Lumley 2007; Naumova et al. 2001). According to 180 piecewise analysis, the summary of the breakpoint was found as $15.94 \pm 1.18$. In the current 181 study, hypotension was measured at five-minute intervals, from beginning of the operation $(0$ 182 minute) to the 40th minute of each. We analyzed data using the GEE and GLMM at two time 183 points (minutes 15 and 20 respectively) to decide the break points using the Akaike Information 184 Criterion (AIC), which is a statistic of selecting model within a likelihood based model, and 185 Quasi-likelihood under the independence model Criterion (QIC), which is a statistic for model 186 selection for GEE models and analogous to AIC. Since the GEE method is a non-likelihood 187 based, AIC is not used for GEE models.

188 When we compared the two models using the 20th minute time point and the 15th minute time 189 point, better results were observed at 20 minutes. For the GEE model, QIC values were 1712.47 for 15 minutes and 1707.50 for the 20th minute. For the GLMM model, AIC values were 1137.56 for the 15th minute, and 1131.10 for the 20th minute. According to these two criteria, the model with the smaller statistic (AIC/QIC) is preferred. Therefore, we defined the cut off point for our study as the 20th minute. Figure $2 \mathrm{~A}$ shows the estimated values of breakpoint according to piecewise regression analysis. Figure 2B shows the breakpoint as the 20th minute, and using the piecewise analysis, two straight lines for each patient were connected at the time of surgery.

Figure 2

\section{Comparison of the Methods using Anesthesiology Data}

198

199

200

201

202

203

204

205

206

207

208

209
The marginal model was applied to the dataset with the GEE approach and the random effects model with GLMM approach. We used SAS (Version 9.2) procedures GENMOD with an independent working correlation matrix and GLIMMIX. In the GLMM, three random effects were determined: the random intercept, and two random slopes (time before and time after). For the two approaches, we tested the covariates and interaction terms. The interaction terms were removed from the models as they were non-significant. The results of comparison between the two models are shown in Table 3.

(Table 3)

The results were similar for the 2 models. Although the parameters that include time-before, time-after, age, and DBP were common in both models, the parameter estimates were different. Differences between regression coefficients and between standard errors from marginal and random effects model are expected. The coefficients estimates from the GEE are lower in 
210

211

212

213

214

215

216

217

218

219

220

magnitude than corresponding coefficients estimates from the GLMM (Fitzmaurice G 2011) except time-after, Marcain-Heavy, Fentanyl. Furthermore, standard errors from the GLMM are larger than those from the GEE. The interpretation of coefficients of both models is different. When looking at table 3 , the estimated regression coefficient of the marginal model corresponding to time-before suggests that the log odds of a hypotension increase 0.0732 unit from the baseline to 20 minutes at every 5 minute interval. The effect of gender (not significant) increased the logit of the probability of hypotension in the population of women more than in the population of men.

$$
\operatorname{logit}\left\{E\left(Y_{i j}\right)\right\}=\beta_{1}+\beta_{2} \text { timebefore }_{i j}+\beta_{3} \text { timeafter }_{i j}+\beta_{4} \text { Age }_{i}+\beta_{5} \text { inder }_{i}+\beta_{6} \text { Operatior }
$$

On the other hand, the result of the random effect model corresponding to time-before suggests that the $\log$ odds of probability of the hypotension for a patient increase 0.1002 units from the baseline to 20 minutes at every 5 minute interval.

$$
\operatorname{logit}\left\{E\left(Y_{i j} \mid b_{i}\right)\right\}=\beta_{1}+\beta_{2} \text { timebefore }_{i j}+\beta_{3} \text { timeafter }_{i j}+\beta_{4} \text { Age }_{i}+\beta_{5} \text { Gender }_{i}+\beta_{6} \text { Op }
$$

221 Comparison of the two estimated coefficients for time-before, $e^{\widehat{\beta_{1}}}=1.08$ and $e^{\widehat{\beta_{1}^{i}}}=1.11$, 222 respectively, from marginal and random effects models clearly show the distinction between 223 these two methods.

224

225

226

227

228

229

230

231

232

233

234

235

236

237

238

239

240

241
Marginal models take into account the averaged relationship, but the random effects models express the relationships on inter-individual via random effects. In our study, although the results were similar, the estimates from the two models were different. The differences between parameter estimates of the two models largely depend on the between-individual heterogeneity. This heterogeneity can be described by random intercept and random slopes (time-before, and time-after) variances in the random model. The random intercept variance is 10.085 , which is very high; this value indicates that there is great importance in between-patient variability in the propensity for hypotension, and it shows that within-subject association is strong. Approximately $95 \%$ of patients have a baseline risk of hypotension that varies from $0.03 \%$ to $99 \%$. The random slopes variances are, respectively 0.003 and 0.007 . Similarly, the $95 \%$ intervals of the random slopes variability vary respectively from $50 \%$ to $55 \%$ and from $47 \%$ to $55 \%$. These values show the amount of variability in the slopes across patients. This inter-individual heterogeneity shows the differences between the parameters estimate of the marginal model and the random effects model.

\section{Discussion}

In longitudinal studies, repeated measures are correlated data that is taken from the same person at different times, and this correlation is important for analysis methods. There are various methods that have been proposed for the analysis of repeated binary data. The GEE, which is a 
242

243

244

245

246

247

248

249

250

251

252

253

254

255

256

257

258

259

260

261

262

263

264

265

266

267

268

269

270

271

272

273

274

275

276

277

278

279

280

281 marginal model, and the GLMM, which is a random effects model, are the two major and most common methods for analyzing such cases.

In general, parameter estimates and standard errors from random effect models are greater than those from marginal models. The difference in the estimates between these two models is due to the correlation between repeated measures. The interpretation of covariates in random effects models is more difficult than in marginal models. For random effect models, the interpretation of estimates is related to changes within subjects. However, marginal models ignore such changes within subjects. This is due to the fact that the target of marginal models is the population, while the target of random effect models is the subject.

In marginal models, the regression coefficient describes how the average rates for any variable may be changed in the study population. The exponential of an estimate parameter represents a population-averaged odds ratio for the response and relates to the sub-population that includes the covariate concerning the sub-population not including the covariate. In the random models, the regression coefficient describes how the odds of any variable for any patient are subject to change. The exponential of the estimate parameter is an odds ratio for a person that has a covariate, when compared to the same person not having a covariate (Fitzmaurice et al. 2004; Hubbard et al. 2010).

The strength of this study lies in the longitudinal nature of the data set. Nevertheless, there were certain limitations to analyzing both methods. The GEE method is not difficult to apply and is now available in the major statistical analysis packages, but the procedures are more complex for the GLMM. What's more, the GEE model does not allow for assessing the suitability of fit (Odueyungbo et al. 2008), whereas the GLMM does (Moscatelli et al. 2012).

Marginal models are popular for binary longitudinal data. However, the choice of method, GEE or GLMM, depends on the aim of the particular study. Marginal models are appropriate if the research focus is on population-average, but if it focuses on individual differences, random effect models are appropriate.

In GLMMs, the fixed effects parameters $\quad \beta$ have conditional interpretations, given the random effects. There are two types of fixed effects. The first of these is that the effect of an explanatory variable refers to the effect on the response of a within-cluster, or within-subject (i.e. subjectspecific) 1-unit increase of that predictor. The other is that the effect of an explanatory variable is a between-cluster. It is in this sense that random effects models are conditional models, as both within- and between-cluster effects apply conditionally on the random effect value (Agresti 2002). On the other hand, effects in marginal models refer to overall clusters (i.e. populationaveraged). For the logit model, the difference between the two models is that the populationaveraged effects are smaller than the cluster-specific effects (Agresti 2002). There are approximate relationships between estimates from these two models in the logistic-normal case. The effect in the marginal model multiplies that of the conditional model by about c (Zeger et al. $1988)$; it is typically smaller in absolute value. The discrepancy increases as $\sigma$ increases (Agresti 2002).

Figure 3 
282

283

284

285

286

287

288

289

290

291

292

293

294

295

296

297

298

299

300

301

302

303

304

305

306

307

308

309

310

311 The authors are most grateful to Prof. Nan Laird for her valuable suggestions regarding statistical 312

Figure 3 shows that when the marginal effect was compared to the subject-specific effect, the marginal effect is smaller than the random effects. For a single explanatory variable and some subjects, the figure shows subject specific curves for $P\left(Y_{i t}=1 \mid b_{i}\right)$ when considerable heterogeneity exists. This corresponds to a relatively large $\sigma$ for the random effects. At any fixed value of the explanatory variable, variability occurs in the conditional means $E\left(Y_{i t} \mid b_{i}\right)=P\left(Y_{i t}=1 \mid b_{i}\right)$. The average of these is the marginal mean, $E\left(Y_{i t}\right)$ (Agresti 2002). Focusing on the range of probability of hypotension between 0.2 and 0.8 , the populationaveraged effect, that is the logistic curve, is more linear, but the slope curves of subject-specific curves rise more rapidly than the marginal slope of probabilities.

\section{Conclusion}

In clinical researches, longitudinal studies with binary repeated events are frequently undertaken. Nevertheless, traditional analyses are inefficient for such studies, and the selection of a more efficient model, namely marginal models or random effects models, has been the primary focus of this study. As is shown, marginal models and random effect models are useful for longitudinal data.

In this study, we compared both methods and found that the regression parameters from GEE are smaller than those from GLMM, while all except three variables and all standard errors from GEE are smaller than those from GLMM.

The individual characteristics of each patient given spinal anesthesia are valuable in terms of understanding the change of probability of hypotension. The identification factors associated with hypotension during anesthesia, such as the type and position of surgery, and the anesthesia drugs and doses, change vary according to the individual differences of patients. The marginal model, GEE, does not measure the association between the change within-subject covariate and the change in the outcome. For this reason, GLMM appears to be more suitable for the analysis of hypotension during spinal anesthesia.

Ethical approval for the study was obtained from Scientific Research Projects Coordination Unit of Akdeniz University (approval number 01.03.2011/50).

\section{Acknowledgements}

analysis. We also thank to Dr. Hanife K. Kabukcu for her assistance with data collection. 
Agresti A. 2002. Categorical Data Anlaysis. Florida: John Wiley\&Sons.

BÜrŽKOVÁ P, and Lumley T. 2007. Longitudinal data analysis for generalized linear models with follow-up dependent on outcome-related variables. Canadian Journal of Statistics 35:485-500.

Carpenter RL, Caplan RA, Brown DL, Stephenson C, and Wu R. 1992. Incidence and risk factors for side effects of spinal anesthesia. Anesthesiology 76:906-916.

Carriere I, and Bouyer J. 2002. Choosing marginal or random-effects models for longitudinal binary responses: application to self-reported disability among older persons. Bmc Medical Research Methodology 2:15.

Dahmen G, and Ziegler A. 2004. Generalized estimating equations in controlled clinical trials: Hypotheses testing. Biometrical Journal 46:214-232.

Fitzmaurice G LNM, Ware J.H. 2011. Applied Longitudinal Analysis,2nd Edition. Fitzmaurice GM, Davidian M, Verbeke G, and Molenberghs G. 2008. Longitudinal Data Analysis. Florida: Chapman and Hall/CRC.

Fitzmaurice GM, and Laird NM. 1993. A Likelihood-Based Method for Analyzing Longitudinal Binary Responses. Biometrika 80:141-151.

Fitzmaurice GM, Laird NM, and Rotnitzky AG. 1993. Regression-Models for Discrete Longitudinal Responses. Statistical Science 8:284-299.

Fitzmaurice GM, Laird NM, and Ware JH. 2004. Applied Longitudinal Analysis New Jersey: John Wiley \& Sons Inc.

Hardin JW, and Hilbe JM. 2003. Generalized Estimating Equations: Boca Raton, FL: Chapman \& Hall/CRC.

Hardin JW, and Hilbe JM. 2007. Generalized Estimating Equations. Wiley Encyclopedia of Clinical Trials: John Wiley \& Sons, Inc.

Hartmann $B$, Junger $A$, Klasen J, Benson $M$, Jost $A$, Banzhaf $A$, and Hempelmann G. 2002. The incidence and risk factors for hypotension after spinal anesthesia induction: an analysis with automated data collection. Anesth Analg 94:1521-1529, table of contents.

Hubbard AE, Ahern J, Fleischer NL, Van der Laan M, Lippman SA, Jewell N, Bruckner T, and Satariano WA. 2010. To GEE or not to GEE: comparing population average and mixed models for estimating the associations between neighborhood risk factors and health. Epidemiology 21:467474.

Katrien A, and Jan B. 2005. Applications of generalized linear mixed models in actuarial statistics. Insurance Mathematics \& Economics 37:374-374.

Kopcke W, Ahrens S, Eisenacher M, and Seuchter S. 2004. Mathematicalstatistical concepts for modelling and prediction of longterm follow-up. Pharmacopsychiatry 37:S98-S102.

Laird NM, and Ware JH. 1982. Random-Effects Models for Longitudinal Data. Biometrics 38:963-974.

Liang KY, and Zeger SL. 1986. Longitudinal Data-Analysis Using Generalized Linear-Models. Biometrika 73:13-22.

Lin CS, Chiu JS, Hsieh MH, Mok MS, Li YC, and Chiu HW. 2008. Predicting hypotensive episodes during spinal anesthesia with the application of 

artificial neural networks. Comput Methods Programs Biomed 92:193197.

Maxson LH. 1933. Spinal Anesthesia: Its Technique, Records, and Results. Cal West Med 39:292-297.

Mccullagh P, and Nelder JA. 1989. Generalized Linear Models. London: Chapman and Hall.

Moscatelli A, Mezzetti M, and Lacquaniti F. 2012. Modeling psychophysical data at the population-level: The generalized linear mixed model. J Vis 12.

Naumova EN, Must A, and Laird NM. 2001. Tutorial in Biostatistics: Evaluating the impact of 'critical periods' in longitudinal studies of growth using piecewise mixed effects models. International Journal of Epidemiology 30:1332-1341.

Odueyungbo A, Browne D, Akhtar-Danesh N, and Thabane L. 2008. Comparison of generalized estimating equations and quadratic inference functions using data from the National Longitudinal Survey of Children and Youth (NLSCY) database. Bmc Medical Research Methodology 8.

Omar RZ, Wright EM, Turner RM, and Thompson SG. 1999. Analysing repeated measurements data: a practical comparison of methods. Statistics in Medicine 18:1587-1603.

Sharma SK, Gajraj NM, and Sidawi JE. 1997. Prevention of hypotension during spinal anesthesia: a comparison of intravascular administration of hetastarch versus lactated Ringer's solution. Anesth Analg 84:111-114.

Tarkkila PJ, and Kaukinen S. 1991. Complications during spinal anesthesia: a prospective study. Reg Anesth 16:101-106.

Warton DI. 2011. Regularized Sandwich Estimators for Analysis of HighDimensional Data Using Generalized Estimating Equations. Biometrics 67:116-123.

Zeger SL, Liang KY, and Albert PS. 1988. Models for longitudinal data: a generalized estimating equation approach. Biometrics 44:1049-1060. 
Table $\mathbf{1}$ (on next page)

Descriptive statistics of dose of anesthetic drugs 


\begin{tabular}{lcccccc}
\hline Anesthetic drugs & Min. & Median & IR & Mean & SD & Max. \\
\hline Marcain-heavy & 0 & 9 & 12 & 7.11 & 6.18 & 25 \\
Chirocaine & 0 & 0 & 13 & 5.29 & 7.89 & 75 \\
Midazolam & 0 & 1 & 1 & 0.82 & 1.00 & 8 \\
Fentanyl & 0 & 0 & 0.05 & 0.03 & 0.06 & 0.20 \\
\hline IR=Interquartile range & & & & & &
\end{tabular}

$\mathrm{IR}=$ Interquartile range 
Table 2 (on next page)

Descriptive statistics of DAP and Pulse by time 


\begin{tabular}{|c|c|c|c|c|}
\hline & \multicolumn{2}{|c|}{ DAP } & \multicolumn{2}{c|}{ Pulse } \\
\hline & Mean \pm SD & Median (IR) & Mean \pm SD & Median (IR) \\
\hline Baseline & $83.82 \pm 14.06$ & $80(20)$ & $87.54 \pm 15.93$ & $85(22)$ \\
\hline 5 minute & $78.58 \pm 13.36$ & $78(20)$ & $84.11 \pm 14.92$ & $80(20)$ \\
\hline 10 minute & $76.18 \pm 13.56$ & $75(20)$ & $82.46 \pm 14.37$ & $80(22)$ \\
\hline 15 minute & $75.65 \pm 13.45$ & $75(20)$ & $81.57 \pm 14.28$ & $80(20)$ \\
\hline 20 minute & $75.24 \pm 12.98$ & $75(20)$ & $80.94 \pm 14.40$ & $80(20)$ \\
\hline 25 minute & $75.08 \pm 12.54$ & $75(18)$ & $80.53 \pm 14.40$ & $80(20)$ \\
\hline 30 minute & $74.50 \pm 12.21$ & $75(15)$ & $79.99 \pm 13.98$ & $80(20)$ \\
\hline 35 minute & $74.19 \pm 12.08$ & $75(15)$ & $79.53 \pm 13.76$ & $80(20)$ \\
\hline 40 minute & $73.49 \pm 12.12$ & $75(15)$ & $78.94 \pm 13.67$ & $78(20)$ \\
\hline
\end{tabular}


Table 3(on next page)

The results of Marginal Model and Random-effects models for data 


\begin{tabular}{|l|ccc|ccc|}
\hline \multicolumn{3}{|c}{ GEE } & \multicolumn{3}{c|}{ GLMM } \\
\hline Intercept & Estimate & SE & p-value & Estimate & SE & p-value \\
Time-before & 0.3949 & 1.8768 & 0.8333 & 0.6016 & 3.1718 & 0.8497 \\
Time-after & 0.0732 & 0.0116 & $\mathbf{< . 0 0 0 1}$ & 0.1002 & 0.0253 & $<.0001$ \\
Age (year) & 0.0428 & 0.0062 & $<. .0001$ & 0.0323 & 0.0165 & $\mathbf{0 . 0 5 0 0}$ \\
Gender (Female) & 0.0271 & 0.0091 & $\mathbf{0 . 0 0 3 0}$ & 0.0469 & 0.0235 & $\mathbf{0 . 0 4 6 3}$ \\
Operation (Urology) & 0.4146 & 0.5646 & 0.4628 & 1.4871 & 1.2435 & 0.2319 \\
Operation (O\&G) & 0.2770 & 0.4535 & 0.5413 & 1.4801 & 1.4207 & 0.2976 \\
Position (Supine) & 0.6551 & 0.7203 & 0.3630 & 1.3287 & 1.0218 & 0.1936 \\
ECG (Normal) & 0.4013 & 0.3366 & 0.2331 & 0.7643 & 0.6850 & 0.2646 \\
DBP & -0.3070 & 0.7932 & 0.6988 & -0.5147 & 1.7085 & 0.7633 \\
Pulse & -0.0863 & 0.0134 & $<. .0001$ & -0.1941 & 0.0224 & $<.0001$ \\
Marcain-Heavy & 0.0041 & 0.0096 & 0.6674 & 0.0244 & 0.0157 & 0.1204 \\
Chirocaine & -0.0028 & 0.0304 & 0.9264 & 0.0008 & 0.0702 & 0.9907 \\
Fentanyl & -0.0297 & 0.0238 & 0.2120 & -0.0488 & 0.0549 & 0.3734 \\
Midazolam & 0.2940 & 2.1471 & 0.8911 & 0.2909 & 4.7399 & 0.9511 \\
& 0.1199 & 0.0964 & 0.2137 & 0.3738 & 0.2474 & 0.1309 \\
\hline
\end{tabular}


Figure 1

Probability of hypotension by time period

Each data point is shown as probability of hypotension in this graph. This plot reveals the variability in subject's hypotension level at entry, hypotension level at exit.

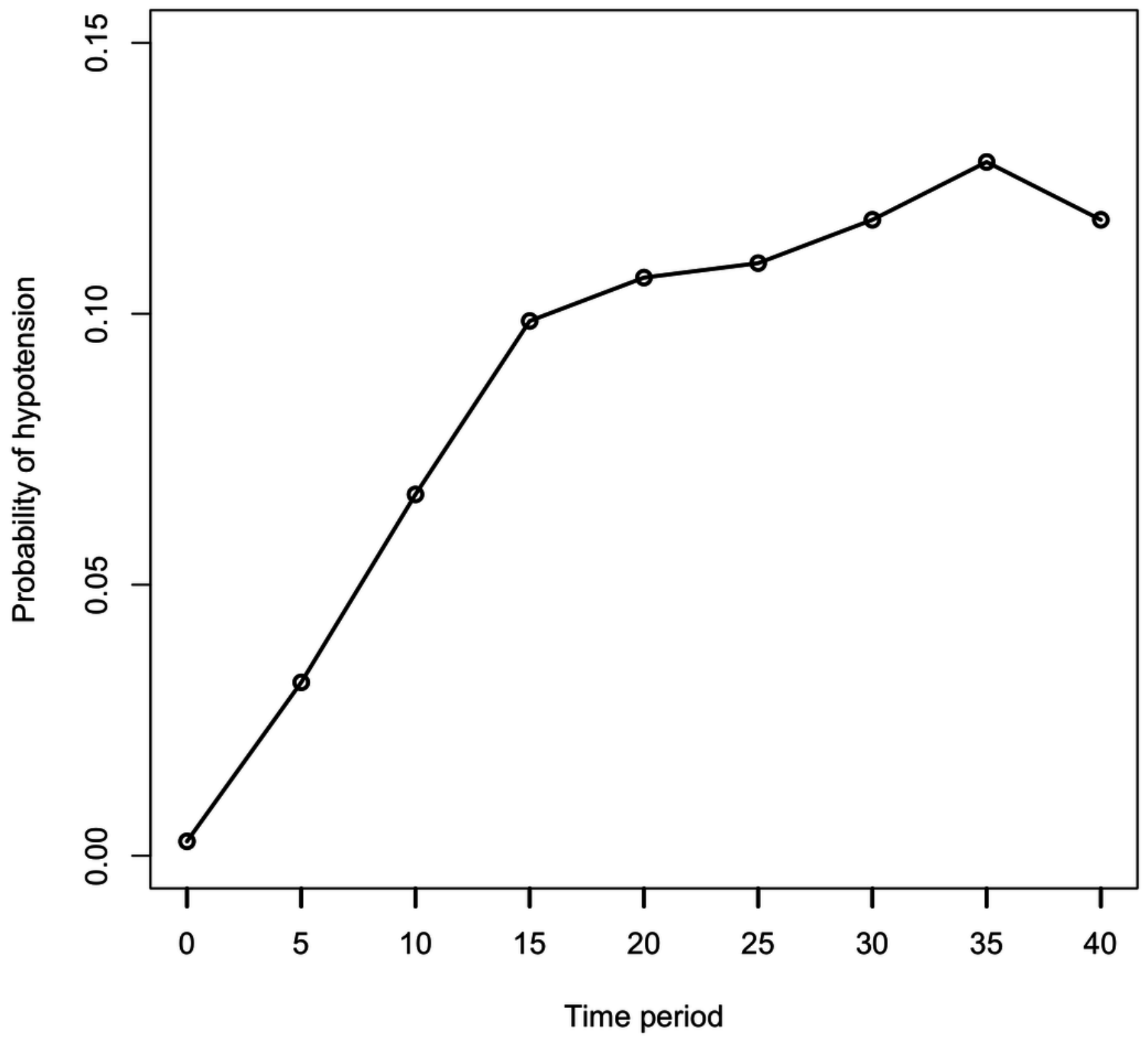


Figure 2

The piecewise regression fit between time and probability of hypotension

(A) This figure shows that the red trend line is calculated with piecewise linear regression analysis with breakpoint. The blue line shows the estimated breakpoint according to piecewise regression. (B) The breakpoint is defined as 20th minute. The line has an increasing trend also after 20th minute. 


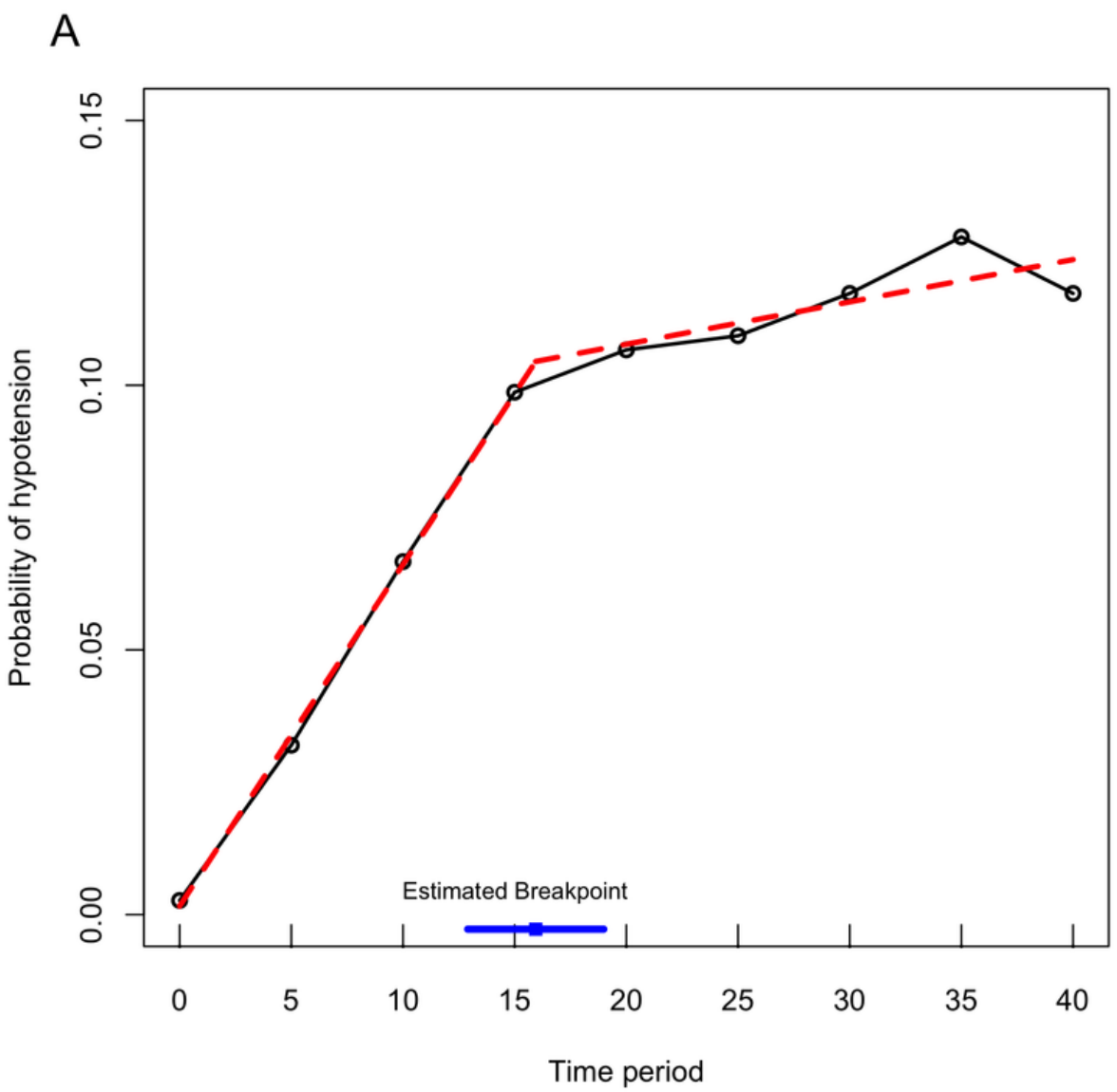

B

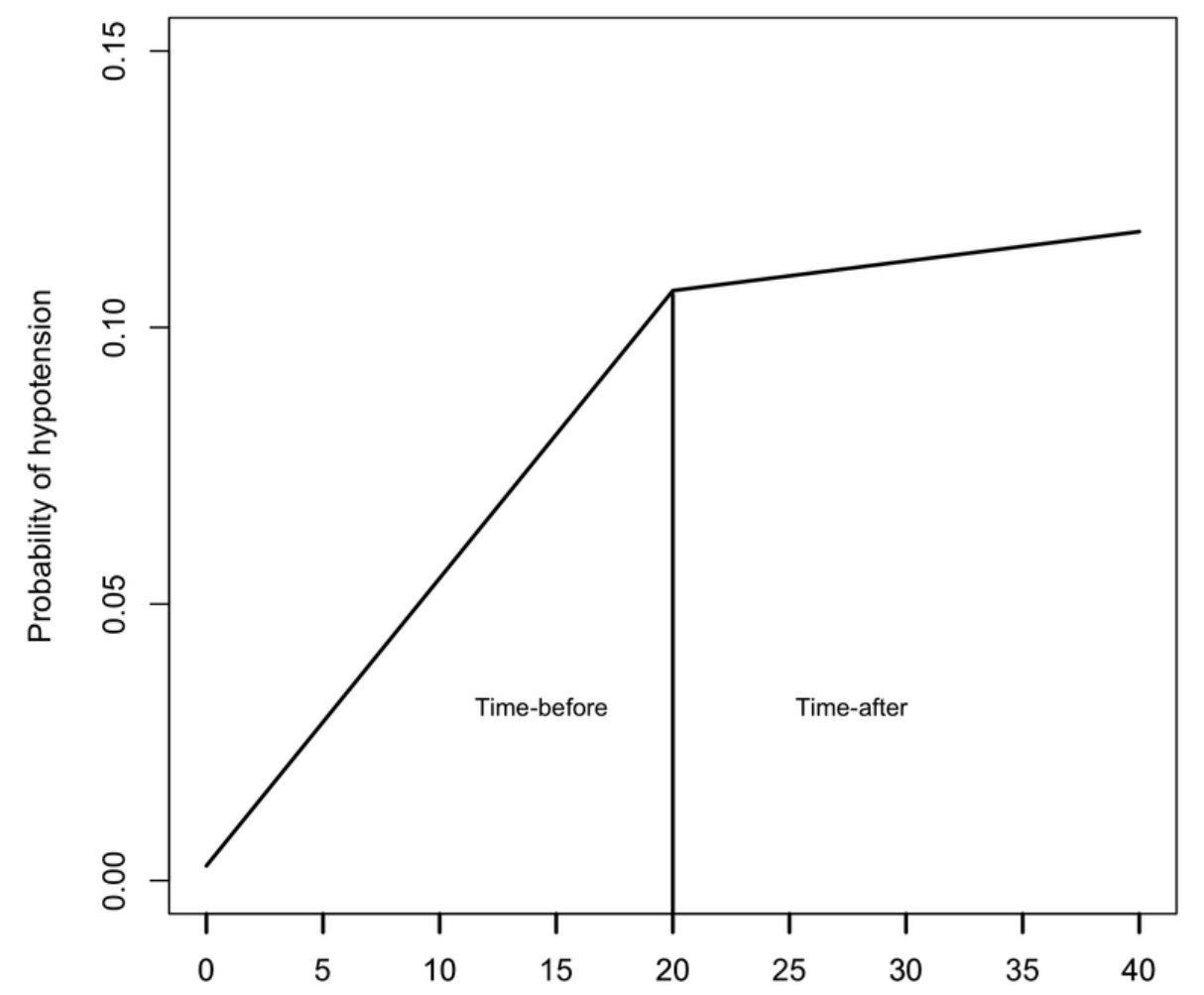

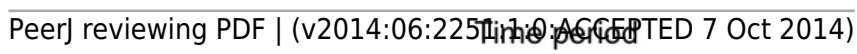


Figure 3

Comparison of random effects model and marginal model

In this figure, the conditional of probabilities of hypotension (dotted lines) and marginal probability of hypotension (solid line) are compared for a single explanatory variable, and for several subjects.

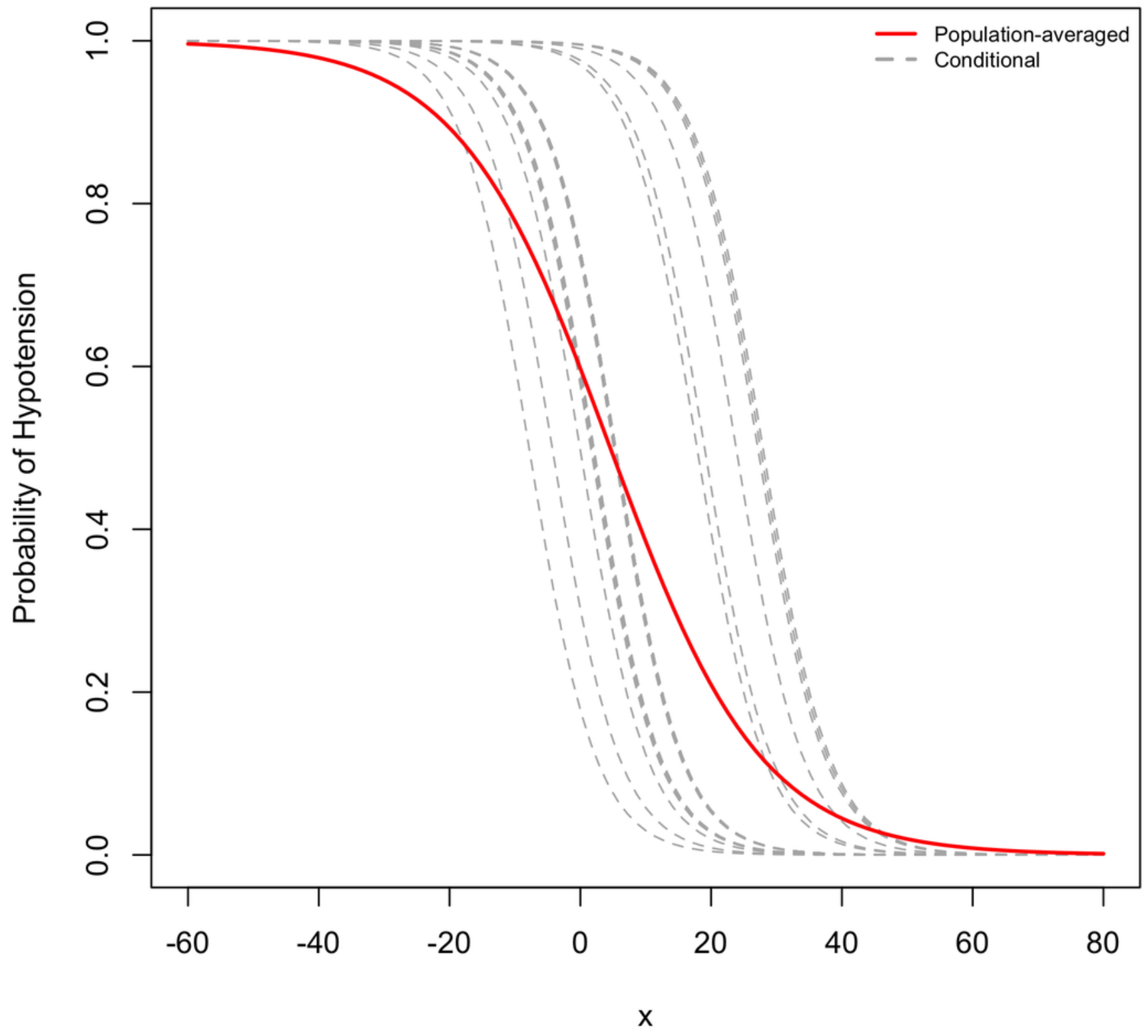

